\title{
Magnetic turbulence in clusters of galaxies
}

\author{
T. A. Enßlin ${ }^{1}$, T. Clarke $^{2}$, C. $\operatorname{Vogt}^{3}$, A. Waelkens ${ }^{1}$, and A. A. \\ Schekochihin $^{4}$ \\ ${ }^{1}$ Max-Planck-Institute for Astrophysics, Karl-Schwarzschild-Str.1, 85741 Garching, Germany. \\ ensslin@mpa-garching.mpg. de \\ ${ }^{2}$ Interferometrics Inc., Naval Research Lab., 4555 Overlook Ave. SW, Washington, DC USA. \\ ${ }^{3}$ Stichting ASTRON, P.O.Box 2,7990 AA Dwingeloo, The Netherlands. \\ ${ }^{4}$ Rudolf Peierls Centre for Theoretical Physics, University of Oxford, 1 Keble Road, Oxford \\ OX1 3NP, UK
}

\begin{abstract}
Galaxy clusters are large laboratories for magnetic plasma turbulence and therefore permit us to confront our theoretical concepts of magnetogenesis with detailed observations. Magnetic turbulence in clusters can be studied via the radio-synchrotron emission from the intra-cluster medium in the form of cluster radio relics and halos. The power spectrum of turbulent magnetic fields can be examined via Faraday rotation analysis of extended radio sources. In case of the Hydra A cool core, the observed magnetic spectrum can be understood in terms of a turbulence-mediated feedback loop between gas cooling and the jet activity of the central galaxy. Finally, methods to measure higher-order statistics of the magnetic field using Stokes-parameter correlations are discussed, which permit us to determine the power spectrum of the magnetic tension force. This fourth-order statistical quantity offers a way to discriminate between different magnetic turbulence scenarios and different field structures using radio polarimetric observations.
\end{abstract}

Keywords. galaxies: clusters: general, magnetic fields, turbulence

\section{How can we compare turbulence models to observations?}

Clusters of galaxies are excellent laboratories for studying turbulent magnetic fields via polarized radio synchrotron emission of relativistic electron populations, which can be found predominately in merging clusters, like Abell 2256 (Fig. 1). But also non-merging cluster exhibit turbulent magnetic fields with Kolmogorov-like power spectra, as an analysis of a Faraday rotation map of the cool core region of the Hydra A cluster shows (also Fig. 1). This turbulence is at least partly stirred by the buoyant motion of radio gas bubbles from the feedback of a central AGN into cooling cluster gas.

Also higher order statics of the magnetic fields may be measurable in future via radio observations. Fig. 2 shows two magnetic field scenarios with identical power spectra, one MHD simulation, and one solenoidal Gaussian random field. Fig. 3 shows their magnetic and tension force power spectra; the former being identical, whereas the latter discriminates the two scenarios. The error bars show how well this could be measured using the Stokes correlators method developed by Waelkens et al. (2009). 

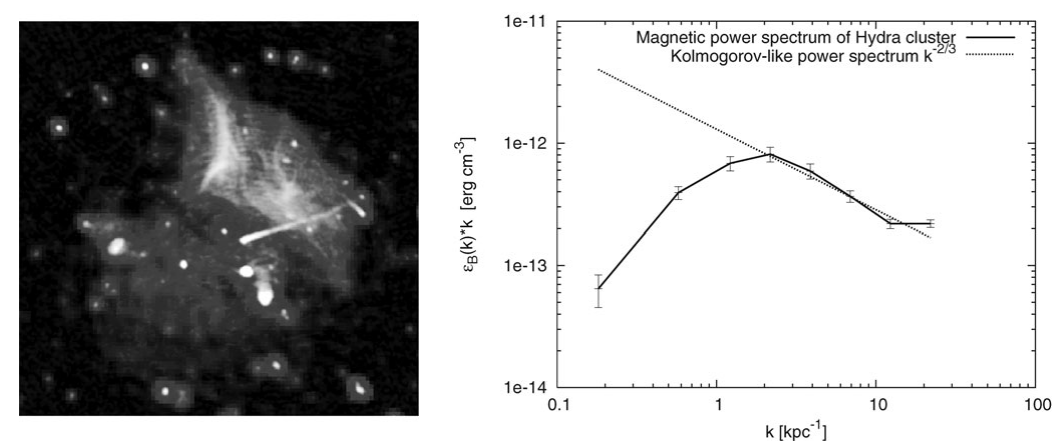

Figure 1. Left: Diffuse radio emission of Abell 2256. A Mpc-sized radio relic marks the probable location of a cluster merger shock wave (top-right structure), and a radio halo indicates the existence of turbulent magnetic fields in the cluster center (roundish central object). From Clarke \& Enßlin (2006) Right: The magnetic power spectra of the cool core of the Hydra cluster by Vogt \& Enßlin (2005).

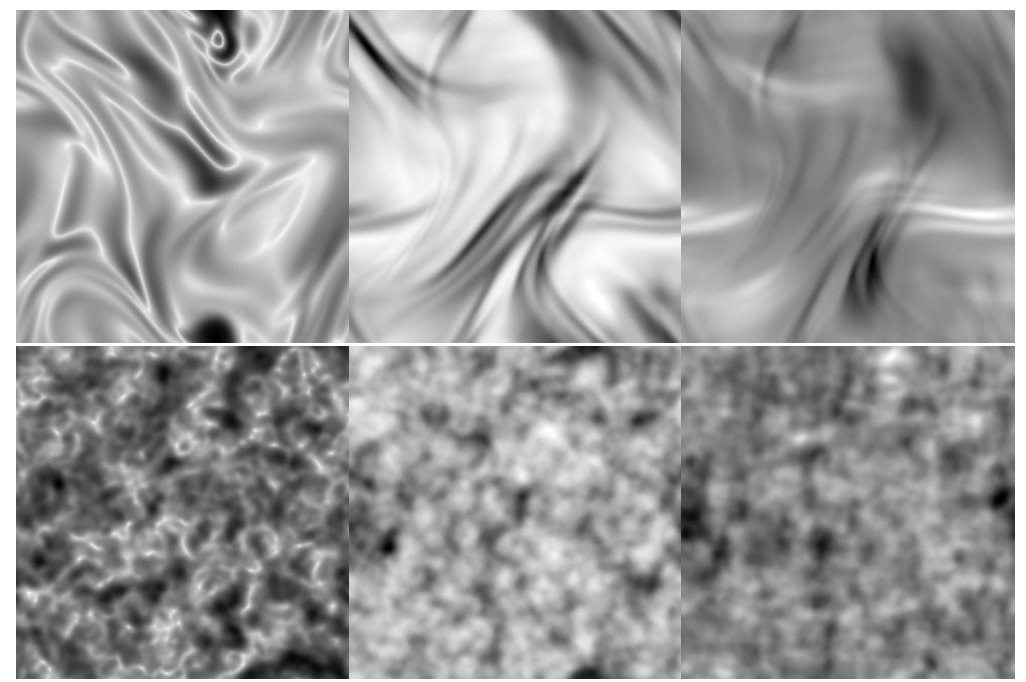

Figure 2. MHD Simulation (top, Schekochihin et al. (2004)) and Gaussian random (bottom). Left: Magnetic energy density. Middle: Total synchrotron intensity Right: Stokes Q synchrotron polarization.
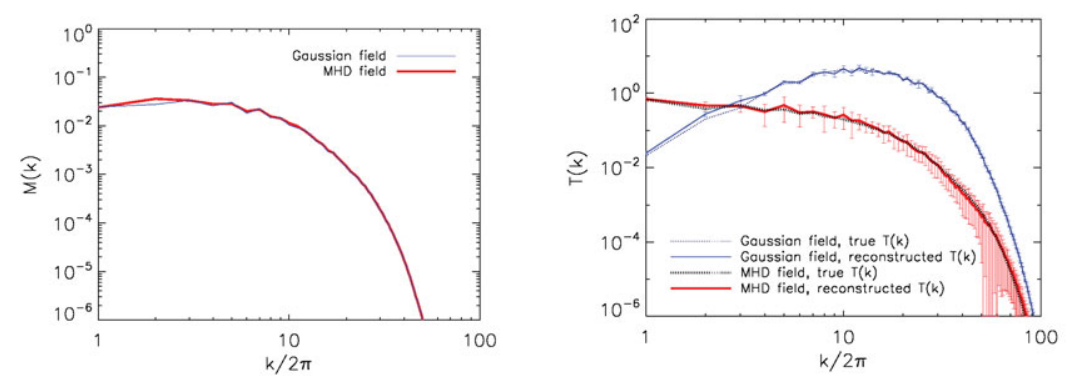

Figure 3. The magnetic power spectra of the two cases are similar (left), although their magnetic tension force power spectrum differ substantially, as revealed by Stokes-correlators (right). 


\section{References}

Clarke, T. E. \& Enßlin, T. A. 2006, AJ, 131, 2900

Schekochihin, A. A., Cowley, S. C., Taylor et al. 2004, ApJ, 612, 276

Vogt, C. \& Enßlin, T. A. 2005, A\&A, 434, 67

Waelkens, A., Schekochihin, A. A., \& Enßlin, T. A. 2009, MNRAS398, 1970 